\title{
Simvastatin enhances the radiosensitivity of p53-deficient cells via inhibition of mouse double minute 2 homolog
}

\author{
JI YOUNG LEE ${ }^{1,3}$, MI-SOOK KIM ${ }^{2}$, JAE EUN JU ${ }^{1,3}$, MI SO LEE ${ }^{1}$, \\ NAMHYUN CHUNG ${ }^{3}$ and YOUN KYOUNG JEONG ${ }^{1}$ \\ ${ }^{1}$ Radiation Non-Clinical Center, ${ }^{2}$ Department of Radiation Oncology, \\ Korea Institute of Radiological and Medical Sciences; ${ }^{3}$ Department of Biosystems Engineering, \\ College of Life Sciences and Biotechnology, Korea University, Seoul, Republic of Korea
}

Received August 22, 2017; Accepted October 21, 2017

DOI: $10.3892 /$ ijo.2017.4192

\begin{abstract}
Simvastatin exhibits anticancer activities, but its molecular mechanisms and radiosensitizing effects relative to p53 status remain unclear. In this study, we investigated whether the combination of simvastatin and ionizing radiation (IR) would enhance the antitumor effects of IR alone in HCT116 p53 $3^{+/+}$and p53 $3^{-/}$colon cancer cells. Using colony formation assays and a xenograft mouse model, we found that simvastatin potently stimulated radiosensitization of HCT116 p53 ${ }^{--}$cells and xenograft tumors. The combination of simvastatin with IR decreased G2/M arrest and delayed the repair of IR-induced DNA damage; however, no differences between the

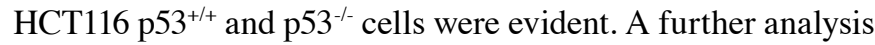
revealed that simvastatin exhibited a novel function, namely, MDM2 suppression, regardless of p53 status. Interestingly, simvastatin induced radiosensitization by enhancing MDM2 suppression and elevating IR-induced p-ATM foci formation compared with IR alone in HCT116 p53 ${ }^{-/}$cells. Furthermore, simvastatin caused accumulations of the FOXO3a, E-cadherin, and p21 tumor suppressor proteins, which are downstream factors of MDM2, in HCT116 p53 $3^{-/}$cells. In conclusion, simvastatin enhanced radiosensitivity by inducing MDM2 inhibition and increasing tumor suppressor protein levels in

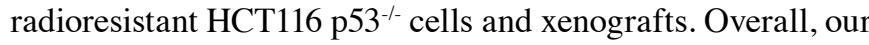
novel findings suggest a scientific rationale for the clinical use of simvastatin as an MDM2 inhibitor and radiosensitizer for p53-deficient colorectal tumor treatments.
\end{abstract}

\section{Introduction}

Drug repositioning is an innovative concept in the drug discovery process whereby new indications that offer the possi-

Correspondence to: Dr Youn Kyoung Jeong, Radiation Non-Clinical Center, Korea Institute of Radiological and Medical Sciences (KIRAMS), 75 Nowon-ro, Nowon-gu, Seoul 01812, Republic of Korea E-mail: amy3523@kirams.re.kr

Key words: simvastatin, radiosensitizer, MDM2 inhibitor, p53, DNA damage repair bility of reduced time and risk for old drugs are sought $(1,2)$. Among them, several drugs to treat metabolic syndrome have been repositioned to treat various cancers. For example, simvastatin is a 3-hydroxy-3-mehtylglutaryl-coenzyme A (HMG-CoA) reductase inhibitor and is often used to treat lipid disorders. Recent studies have shown that simvastatin exhibits anticancer effects by regulating proliferation, apoptosis, and metastasis in various tumors (3-6). Studies that have examined the underlying mechanisms of the anticancer effects indicate that simvastatin inhibits cyclin-dependent kinases (CDK) and cyclins (7) and promotes apoptosis by upregulating the Notch1 gene (8) and inhibiting Akt phosphorylation $(9,10)$. Additionally, simvastatin also enhances radiosensitization by suppressing BIRC5 (survivin) and CTGF (connective tissue growth factor) in gastric cancer and colorectal cancer (11). Furthermore, several studies have shown that simvastatin triggers caspase-dependent apoptosis via p53 activation $(8,12)$, but another study has shown that simvastatin induces apoptosis through the JNK signaling pathway independent of p53 status (13). However, the anticancer mechanism of simvastatin relative to the p53 status remains unclear.

Radiotherapy is a highly effective tool for cancer treatment and is used to treat $>50 \%$ of cancer patients (14). Although several factors such as overexpression of DNA repair protein (15) and tumor microenvironment (16) affect the radiosensitivity, many studies have focused on p53 status correlated with radiation response (17-19). In unstressed cells, p53 is maintained at low levels and controlled by mouse double minute 2 homolog (MDM2), which is a negative regulator of p53 (20). However, upon exposure to ionizing radiation (IR), p53 is phosphorylated and subsequently inhibits the p53-MDM2 interaction, which increases the expression of proteins that induce apoptosis (e.g., bax and bcl-2), cell cycle arrest (e.g., p21), and DNA damage repair (e.g., Rad51) (21). Therefore, p53 is a good prognostic factor of radiosensitivity, and p53 mutations may promote resistance to radiotherapy.

Radioresistant p53-deficient tumors usually express the wild-type MDM2 protein because mutations in both MDM2 and p53 do not typically occur within one tumor (22). Wild-type MDM2 protein may retain its role in cell cycle control, cell survival, and invasion (23). Additionally, MDM2 is frequently overexpressed in advanced cancer patients with 
invasive and high-grade tumors (24). Thus, inhibition of MDM2 may reinforce anticancer activities in p53-deficient cancers. Recently, several studies have shown that MDM2 inhibitors display broad anticancer activity in various cancers. Feng et al reported that MI-219, a small-molecule inhibitor of MDM2, sensitizes prostate cancer to radiotherapy in a p53-dependent manner (25). Another study has demonstrated that p53-independent induction of p21 through MDM2 downregulation might contribute to anticancer activity (26). Furthermore, anti-MDM2 antisense oligonucleotides have chemosensitization and radiosensitization effects in vitro and in vivo in several human cancer models regardless of $\mathrm{p} 53$ status (27). However, the effects of combining the MDM2 inhibitor with radiotherapy in p53-deficient and p53 wild-type cancers remain unclear.

In this study, we investigated whether the combination of simvastatin and IR would radiosensitize p53-deficient cancer cells. Furthermore, by focusing on the MDM2 and p53 interaction, we identified novel functions for simvastatin as an MDM2 inhibitor and defined the impact of simvastatin and MDM2 on cellular radiosensitivity. We hope that our results contribute to a scientific rationale for the clinical use of simvastatin in combination with radiotherapy in patients with malignant cancers, particularly p53-deficient cancers.

\section{Materials and methods}

Materials. HCT116 p53 $3^{+/+}$human colorectal cancer cells were obtained from the Korean Cell Line Bank (Seoul, South Korea), and HCT116 p53 ${ }^{-/}$human colorectal cancer cells were kindly provided by Dr B. Vogelstein of Johns Hopkins University. Simvastatin was obtained from Sigma-Aldrich Chemical Corp. (St. Louis, MO, USA) and Cayman Chemical Corp. (Ann Arbor, MI, USA). Roswell Park Memorial Institute (RPMI)-media, fetal bovine serum (FBS), trypsin, and antibiotics were purchased from Welgene (Seoul, Korea). Antibodies against MDM2, p53, cyclin D1, survivin, p21, ku70, ku80, DNA-PKcs, p-ATM and GAPDH were purchased from Santa Cruz (San Diego, CA, USA). Cyclin A2, pRb, p-cdc2 (CDK1), CDK2, cyclin B1, Rad50, FOXO3a, and $\mathrm{E}$-cadherin antibodies that were used in this study were obtained from Cell Signaling Technology (Mississauga, ON, Canada). CDK4 and CDK6 antibodies were purchased from Bethyl Laboratories (Montgomery, TX, USA). Rad51 and ERCC1 antibodies were purchased from Abcam Bio (Cambridge, MA, USA) and $\gamma \mathrm{H} 2 \mathrm{AX}$ was purchased from Millipore (Bedford, MA, USA).

Cell culture and treatments. HCT116 p53 $3^{+/+}$and $\mathrm{p} 53^{-/-}$cells were cultured in RPMI-1640 supplemented with $10 \%(\mathrm{v} / \mathrm{v})$ FBS, $100 \mathrm{U} / \mathrm{ml}$ penicillin and $100 \mu \mathrm{g} / \mathrm{ml}$ streptomycin. All cells were cultured in a humidified incubator under an atmosphere of $5 \% \mathrm{CO}_{2}$ at $37^{\circ} \mathrm{C}$. Simvastatin was dissolved in $1 \mathrm{ml}$ of DMSO to a concentration of $12 \mathrm{mM}$ and stored at $4^{\circ} \mathrm{C}$. Cells were treated with the indicated concentrations of simvastatin $24 \mathrm{~h}$ before irradiation.

Irradiation. For in vitro experiments, the cells were irradiated with a ${ }^{137} \mathrm{Cs} \gamma$-ray source (Atomic Energy of Canada, Ltd., Chalk River, Ontario, Canada) at a dose rate of $2.67 \mathrm{~Gy} / \mathrm{min}$.
For in vivo experiments, mice were irradiated using a ${ }^{60} \mathrm{Co} \gamma$-ray source (Theratron 780; Atomic Energy of Canada) with a $0.5 \mathrm{~cm}$ diameter bolus of tissue equivalent material to allow for dose buildup.

Proliferation assays. The cells were seeded in a 96-well plate at a density of $1 \times 10^{3}$ cells per well. Varying concentrations of simvastatin $(0-80 \mu \mathrm{M})$ were added to each well, and the cells were incubated for $48 \mathrm{~h}$, followed by addition of the water-soluble tetrazolium (WST)-1 cytotoxicity assay reagent (EZ-Cytox; Dogen, Seoul, Korea) according to the manufacturer's instructions.

Clonogenic survival assays. Cells were trypsinized, seeded in $4 \mathrm{ml}$ medium and incubated overnight before the simvastatin treatment. Cells were treated with $0-20 \mu \mathrm{M}$ simvastatin for $48 \mathrm{~h}$ or $5 \mu \mathrm{M}$ simvastatin for $24 \mathrm{~h}$ before IR and then further incubated for $24 \mathrm{~h}$. After 12 days, cells were fixed for 30 min with $100 \%$ methanol and stained with $0.4 \%$ crystal violet, followed by rinsing with tap water. Colonies containing $>50$ cells were counted.

Xenograft tumors in athymic mice. Athymic Balb/c nude mice (4-week-old males) were obtained from Nara Biothech Co. (Seoul, Korea) and maintained in a laminar airflow cabinet under specific pathogen-free conditions. HCT116 p53 ${ }^{+/+}$and p53 $3^{-/}$xenograft mouse models were established by subcutaneous injection of $3 \times 10^{6} \mathrm{HCT} 116 \mathrm{p} 53^{+/+}$or $\mathrm{p} 53^{-/-}$cells into the right thigh. When the tumor reached a volume of $\sim 100$ $\mathrm{mm}^{3}$, the mice were randomly divided into four groups $(\mathrm{n}=5)$ : control, simvastatin, IR, and simvastatin plus IR. The simvastatin-treated groups (simvastatin alone and simvastatin plus IR) were injected (intraperitoneally) once per day with $20 \mathrm{mg} /$ $\mathrm{kg}$. When the tumor volume of the control group reached $150-180 \mathrm{~mm}^{3}$, the IR-treated groups (IR alone and simvastatin plus IR) were treated with a single 5 Gy fraction of localregional irradiation using a ${ }^{60} \mathrm{Co}$ irradiator. The tumor volume (V) was calculated using the standard formula: $\mathrm{V}\left(\mathrm{mm}^{3}\right)=\pi / 6$ $\mathrm{x}$ (smaller diameter) ${ }^{2} \mathrm{x}$ (larger diameter). Mice were euthanized by carbon dioxide $\left(\mathrm{CO}_{2}\right)$ inhalation when the average tumor volume of the control group reached $1,000 \mathrm{~mm}^{3}$.

Cell cycle analysis. After simvastatin $(5 \mu \mathrm{M})$ exposure for $24 \mathrm{~h}$, cells were irradiated, incubated for $24 \mathrm{~h}$, harvested, stained with propidium iodide $(1 \mathrm{mg} / \mathrm{ml})$ according to the manufacturer's protocol, and analyzed using a FACSCanto II flow cytometer (Becton-Dickinson, Franklin Lakes, NJ, USA). A minimum of 10,000 cells per sample were counted, and data analysis was performed using the CellQuest software.

Immunofluorescence. Immunofluorescent staining was performed to determine the nuclear distributions of $\gamma \mathrm{H} 2 \mathrm{AX}$ and p-ATM foci in HCT116 p53 ${ }^{+/+}$and $\mathrm{p} 53^{-/-}$cells using image analysis. Cells were grown on chamber slides 1 day prior to the irradiation or simvastatin treatments. After simvastatin $(5 \mu \mathrm{M})$ exposure for $24 \mathrm{~h}$, cells were irradiated and incubated 1 or $24 \mathrm{~h}$ before harvest. Cells were fixed (4\% paraformaldehyde in phosphate-buffered saline, PBS, $10 \mathrm{~min}$ ), permeabilized (0.6\% Triton X-100 in PBS, $10 \mathrm{~min}$ ), and blocked (4\% FBS in PBS, $1 \mathrm{~h}$ ) and then incubated overnight at $4^{\circ} \mathrm{C}$ or for $>4 \mathrm{~h}$ 
A

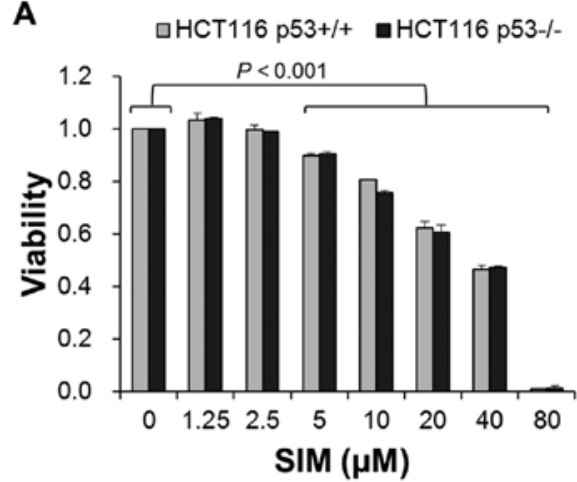

B

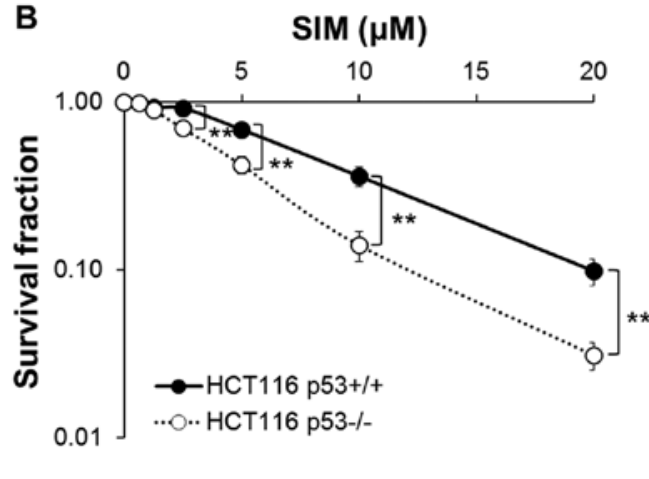

Figure 1. Simvastatin induces higher clonogenic cell death in p53-deficient cells than p53 wild-type cells. HCT116 p53 ${ }^{+/+}$and p53 ${ }^{-/}$cells were treated with vehicle or indicated concentrations of simvastatin (SIM) for $48 \mathrm{~h}$ and then examined using (A) WST-1 assays and (B) clonogenic assays. Statistically significant differences are shown; ${ }^{* *} \mathrm{P}<0.01$ between HCT116 $\mathrm{p} 53^{+/+}$and $\mathrm{p} 53^{-/-}$cells.

at room temperature with primary antibodies (1:500, anti$\gamma \mathrm{H} 2 \mathrm{AX}$, anti-p-ATM). The cells were then washed with PBS and incubated for $1 \mathrm{~h}$ at room temperature in the dark with appropriate fluorescein isothiocyanate (FITC)-labeled secondary antibodies (1:500), including Alexa Fluor 488 goat anti-mouse IgG $(\mathrm{H}+\mathrm{L})$ for $\gamma \mathrm{H} 2 \mathrm{AX}$ and $\mathrm{p}-\mathrm{ATM}$ (green). The cells were then washed with PBS, stained with DAPI (blue), and mounted onto slides using fluorescence mounting medium. The slides were finally examined using a fluorescence microscope with digital imaging system (Olympus, Tokyo, Japan), and images were captured using a charge-coupled device camera. For the quantitative analysis, foci-positive cells were counted in $\geq 50$ cells from randomly captured images.

Western blot analysis. Cells were lysed with a radioimmunoprecipitation assay (RIPA) buffer, and the proteins were separated by sodium dodecyl sulfate (SDS) polyacrylamide gel electrophoresis (PAGE) and transferred to nitrocellulose membranes. The membranes were blocked with $5 \%(\mathrm{v} / \mathrm{v})$ skim milk in PBS with $0.1 \%$ Tween-20, incubated with the indicated primary antibodies (1:1,000) and secondary antibodies (1:1,000), and subsequently developed using an enhanced chemiluminescence western blotting substrate (Cyanangen Srl, Bologna, Italy) using the ImageQuant LAS-4000 mini (GE Healthcare, Fairfield, CT, USA). The signal intensity of each band was measured using the Multigauge V3.0 software (Fujifilm Life Science, Tokyo, Japan).

Statistical analysis. All data are expressed as the mean \pm standard error of the mean (SEM). Statistical analysis was performed using independent t-tests and one-way analysis of variance (ANOVA) followed by Tukey's honest significant difference (HSD) test through the Statistical Package for the Social Sciences (SPSS) software (version 23.0, Chicago, IL, USA). The combination index (CI) was calculated using the formula $\mathrm{CI}=(\% \mathrm{~A} \times \% \mathrm{~B}) /(\% \mathrm{AB} \times 100)$, where $\% \mathrm{~A}$ and $\% \mathrm{~B}$ are the percent viability of simvastatin and IR alone and $\% \mathrm{AB}$ is the combination of them. Subadditivity was defined as $\mathrm{CI}<1.0$; additivity was defined as $\mathrm{CI}, 1.0$; and supra-additivity was defined as CI>1.0 (28).

Ethics statement. All animal study protocols and experiments were approved by the Institutional Animal Care and Use
Committee (IACUC) of the Korean Institute of Radiological and Medical Sciences (KIRAMS 2016-53).

\section{Results}

Simvastatin inhibits cell proliferation in HCT116 p53 wild-type and p53-deficient cells. To investigate the effect of simvastatin on the cell proliferation, HCT116 ${\mathrm{p} 53^{+/+}}^{\text {and }} \mathrm{p} 53^{-1-}$ cells were exposed to various concentrations of simvastatin (0-80 $\mu \mathrm{M})$ for $48 \mathrm{~h}$. Fig. 1A shows that simvastatin inhibited the proliferation of both cells in a concentration-dependent manner. Simvastatin decreased cell proliferation by $89.9 \pm 0.8$, $80.8 \pm 0.0,62.2 \pm 2.6,46.7 \pm 1.3$ and $0.7 \pm 0.2 \%$ in HCT116 $\mathrm{p}^{+/+}$ cells and $90.6 \pm 0.6,75.6 \pm 0.9,60.7 \pm 2.8,47.2 \pm 0.6$ and $1.3 \pm 0.6 \%$ in HCT116 $\mathrm{p53}^{-/}$cells at the concentrations of 5, 10, 20, 40 and $80 \mu \mathrm{M}$, respectively $(\mathrm{P}<0.001)$. In addition, simvastatin weakened cell-cell interaction and cell adherence in both cells (data not shown). However, simvastatin had no differences in the cytotoxicity between HCT116 $\mathrm{p} 53^{+/+}$and $\mathrm{p} 53^{-/-}$cells.

Simvastatin induces lower clonogenic cell survival in HCT116 p53-deficient than p53 wild-type cells. We also assessed p53-dependent clonogenicity by simvastatin in HCT116 $553^{+/+}$ and $\mathrm{p} 53^{-/-}$cells. In HCT116 $\mathrm{p} 53^{+/+}$cells, simvastatin treatment showed clonogenic survival fractions of $68.6 \pm 6.0,36.2 \pm 4.9$ and $9.9 \pm 1.8 \%$ at the concentrations of 5,10 and $20 \mu \mathrm{M}$, respectively $(\mathrm{P}<0.001)$ (Fig. 1B). Moreover, simvastatin induced clonogenic survival fractions of $89.2 \pm 2.0,69.6 \pm 4.0,42.2 \pm 5.1$, $14.1 \pm 2.9$ and $3.1 \pm 0.6 \%$ in HCT116 p53 ${ }^{-/}$cells at the concentrations of $1.25,2.5,5,10$ and $20 \mu \mathrm{M}$, respectively $(\mathrm{P}<0.001)$. Simvastatin significantly decreased clonogenic cell survival of both groups of cells in a concentration-dependent manner, and HCT116 p53 $^{-/}$cells were more sensitive to clonogenic cell death by simvastatin than HCT116 p53 ${ }^{+/+}$cells were $(\mathrm{P}<0.01)$.

Simvastatin enhances the radiosensitivity of HCT116 p53-deficient cells and xenograft tumors. To evaluate the radiosensitization effects of simvastatin in vitro, we treated HCT116 $\mathrm{p} 53^{+/+}$and $\mathrm{p} 53^{-/}$cells with $5 \mu \mathrm{M}$ simvastatin for $24 \mathrm{~h}$ followed by IR and then replaced the medium with fresh medium at $24 \mathrm{~h}$ after IR. In the clonogenic assays, the surviving fractions from 2 to 6 Gy IR were $46.9 \pm 3.4,6.8 \pm 1.2$ and $0.5 \pm 0.1 \%$ and the combinations with simvastatin were $48.2 \pm 3.7,6.2 \pm 1.0$ 


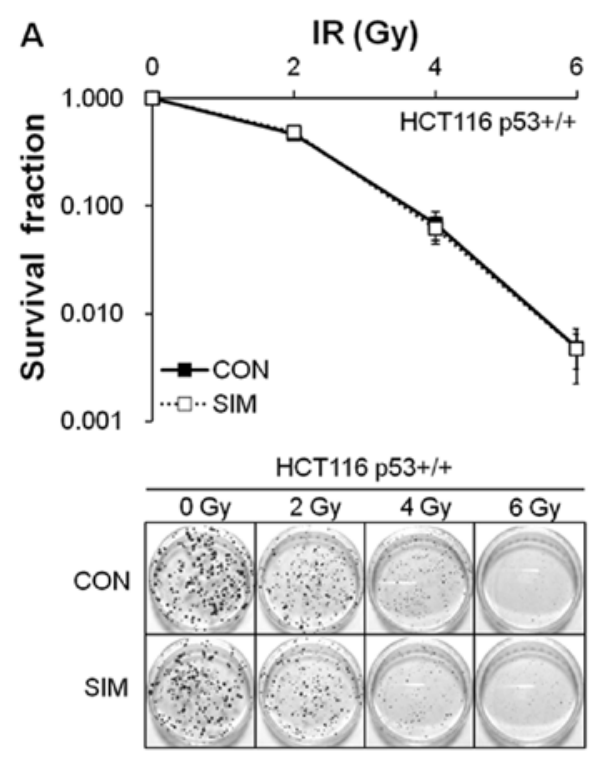

C

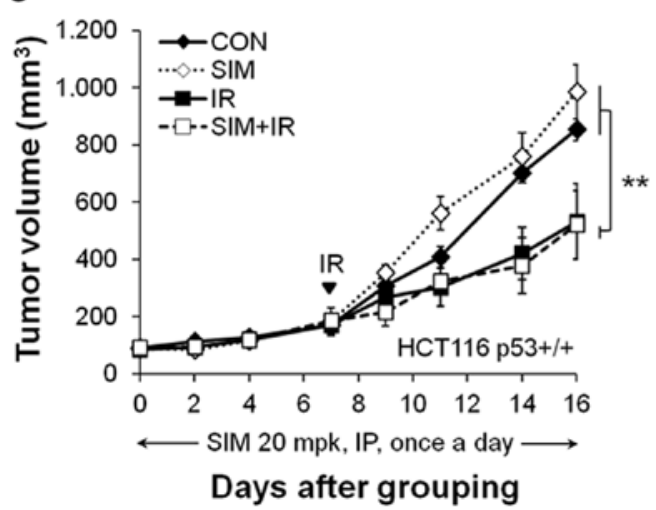

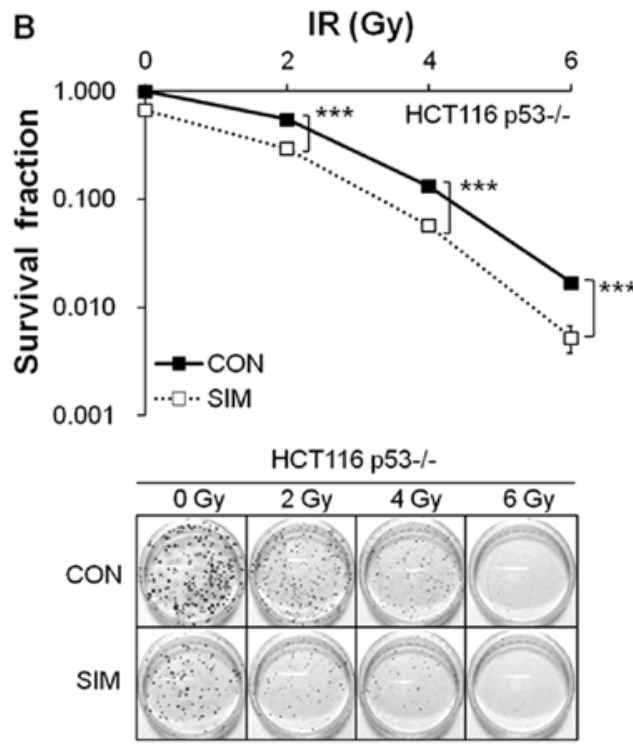

D

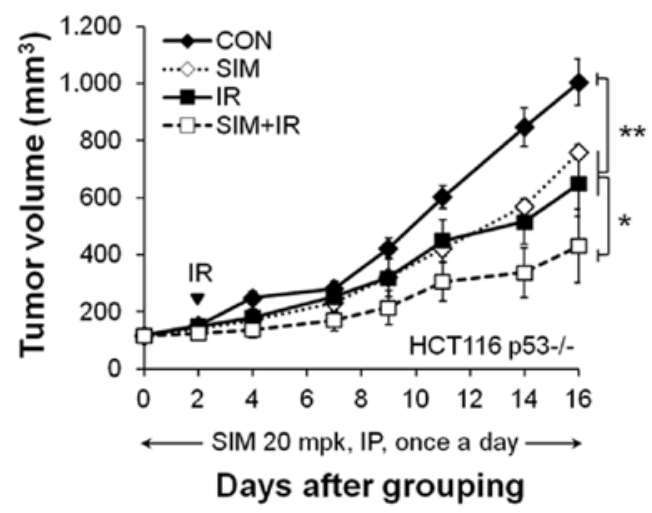

Figure 2. Simvastatin radiosensitizes p53-deficient cells and xenograft tumors. HCT116 p53 $3^{+/+}$(A) and p53 ${ }^{-/-}$(B) cells were pretreated with $5 \mu \mathrm{M}$ simvastatin (SIM) for $24 \mathrm{~h}$, irradiated with various doses of $\gamma$-radiation and measured using clonogenic assays. Nude mice bearing xenograft tumors of HCT116 $53^{+/+}$ and $\mathrm{p} 53^{-/}$cells were treated intraperitoneally with $20 \mathrm{mg} / \mathrm{kg} / \mathrm{day}$ simvastatin for 16 days. Values represent the mean tumor volumes of the HCT116 p53 ${ }^{+/+}(\mathrm{C})$ and $\mathrm{p}^{-/-}$(D) xenograft tumors. Statistically significant differences are shown; ${ }^{*} \mathrm{P}<0.05,{ }^{* * *} \mathrm{P}<0.01,{ }^{* * *} \mathrm{P}<0.001$.

and $0.5 \pm 0.1 \%$ in HCT116 p53 $3^{+/+}$cells (Fig. 2A). In HCT116 $\mathrm{p} 53^{-/-}$cells, the surviving fractions from 2 to 6 Gy IR were $55.1 \pm 1.6,13.3 \pm 0.5$ and $1.7 \pm 0.2 \%$ and the combinations with simvastain were $44.2 \pm 1.4,8.6 \pm 0.7$ and $0.8 \pm 0.1 \%$ (Fig. 2B). Simvastatin plus IR significantly reduced the clonogenic

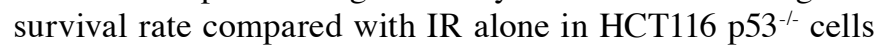
$(\mathrm{CI}>1.0 ; \mathrm{P}<0.01)$ but not in $\mathrm{p} 53^{+/+}$cells. These results showed that the radiosensitizing effect of simvastatin was only shown

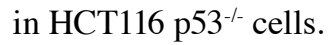

We then investigated whether simvastatin enhanced radiosensitivity in mice xenografted with $\mathrm{HCT} 116 \mathrm{p} 53^{+/+}$ and $\mathrm{p} 53^{-/-}$cells. When the mean tumor volume in the control group reached $\sim 1,000 \mathrm{~mm}^{3}$, simvastatin reduced HCT116 p53 $3^{-/-}$xenograft tumor growth (by $24.4 \pm 2.5 \%, \mathrm{P}<0.01$; compared with control) but did not suppress HCT116 p53 $3^{+/+}$ xenograft tumor growth (Fig. 2C and D). Moreover, simvastatin plus IR enhanced HCT116 p53 $^{-/-}$xenograft tumor growth inhibition more strongly than IR alone (IR, 35.4 $11.2 \%$; simvastatin + IR, $57.2 \pm 12.8 \%, \mathrm{P}<0.05)$ but did not enhance $\mathrm{p} 53^{+/+}$xenograft tumor growth inhibition (IR, 37.5 $\pm 15.4 \%$; simvastatin + IR, $38.8 \pm 14.0 \%$ ). After the injection of simvastatin for 16 days, there was no significant body weight loss in mice treated with simvastatin (20 mg/kg/day) compared with the vehicle-treated control group (data not shown). In summary, simvastatin enhanced radiosensitivity of HCT116 p53 $3^{-/-}(\mathrm{CI}>1.0)$, but not $\mathrm{p} 53^{+/+}$, cells and xenograft tumors.

Simvastatin reduces IR-induced G2/M arrest of HCT116 p53-deficient and p53 wild-type cells. To examine the effect of simvastatin on IR-induced cell cycle progression in the presence or absence of p53, we analyzed cell cycle alterations by flow cytometry. As shown in Fig. 3A, simvastatin markedly attenuated IR-induced G2/M arrest and shifted HCT116 p53 ${ }^{-1-}$ and $\mathrm{p} 53^{+/+}$cells into the $\mathrm{G} 1 / \mathrm{S}$ phases $(\mathrm{P}<0.001)$. Specifically, simvastatin decreased the IR-induced fraction of HCT116 p53 ${ }^{-/-}$cells in the G2/M phase (IR, 76.8 $\pm 1.1 \%$; simvastatin + IR, $29.8 \pm 2.7 \%$ ) compared with the fraction of $\mathrm{p} 53^{+/+}$cells in $\mathrm{G} 2 / \mathrm{M}$

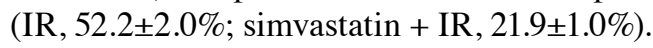

To confirm the decrease in IR-induced G2/M arrest by simvastatin, we evaluated the expression levels of cell cycleregulated proteins, including cyclin-dependent kinases (CDK1, CDK2, CDK4 and CDK6), cyclins (cyclin D1, cyclin A2 and cyclin B1), pRb, and survivin, by western blotting. Fig. 3B shows that simvastatin decreased all cell cycle-regulated 
A
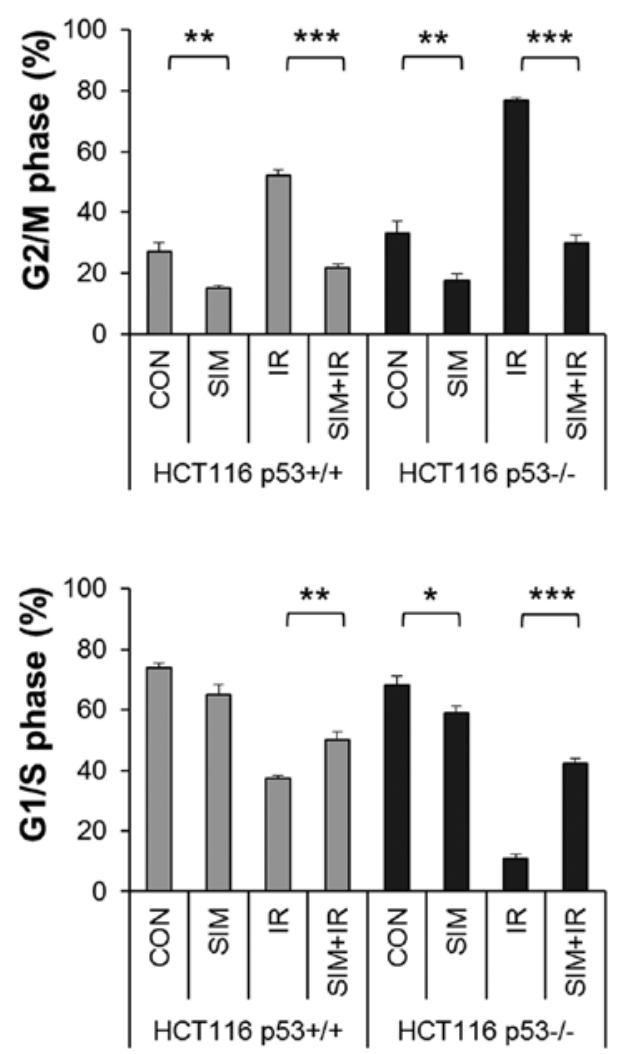

B

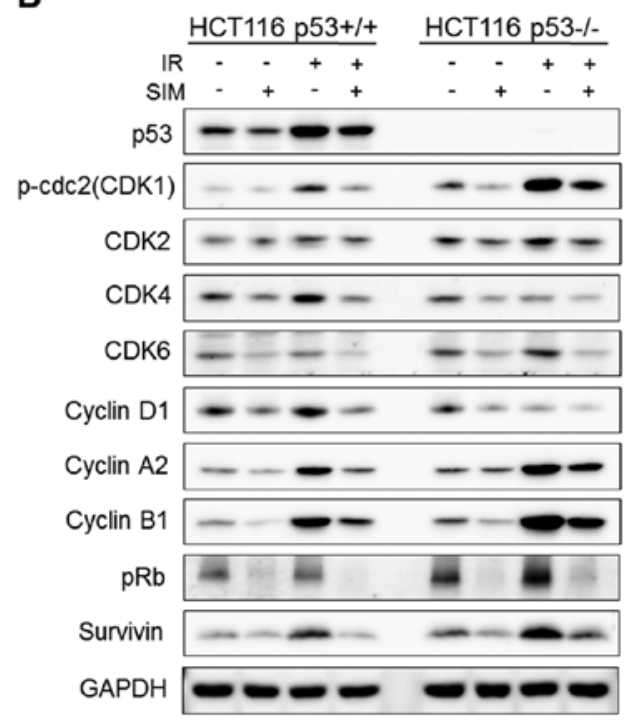

Figure 3. Simvastatin inhibits cell cycle progression by significantly attenuating IR-induced G2/M arrest. HCT116 p53 ${ }^{+/+}$and p53 ${ }^{-/-}$cells were pretreated with $5 \mu \mathrm{M}$ simvastatin (SIM) for $24 \mathrm{~h}$ and then irradiated with $6 \mathrm{~Gy} \mathrm{IR}$. (A) Flow cytometric cell cycle analysis of PI incorporation was performed after treatment of cells with IR for $24 \mathrm{~h}$. Values represent the mean \pm SEM. Statistically significant differences are shown; ${ }^{*} \mathrm{P}<0.05,{ }^{* *} \mathrm{P}<0.01,{ }^{* * *} \mathrm{P}<0.001$. (B) Western blot analysis was performed to determine the protein expression levels of the CDKs (CDK1, CDK2, CDK4 and CDK6), cyclins (cyclin D1, cyclin A2, and cyclin B1), pRb, and survivin in HCT116 $553^{+/+}$and $\mathrm{p} 53^{-/-}$cells following treatment with simvastatin, IR, or both.

proteins that we evaluated. Furthermore, simvastatin plus IR significantly reduced the protein levels that were upregulated after IR (CDK1, CDK2, cyclin A2, cyclin B1 and survivin) in

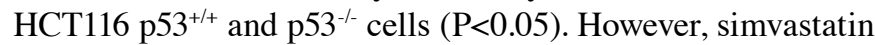
had no differences in the cell cycle-regulated protein levels between the cell lines.

Simvastatin impaires DNA damage repair in HCT116 p53-deficient cells more than in HCT116 p53 wild-type cells. To analyze the effect of simvastatin on DNA damage, we performed immunofluorescence staining of $\gamma \mathrm{H} 2 \mathrm{AX}$, a marker of DNA damage, and visualized the nuclear DNA using DAPI. At $1 \mathrm{~h}$ after IR, simvastatin plus IR exhibited a significantly increased number of $\gamma \mathrm{H} 2 \mathrm{AX}$ foci compared with IR alone in the HCT116 $\mathrm{p}^{\mathrm{H}} 3^{++}$and $\mathrm{p} 53^{-/-}$cells (Fig. 4A). Additionally, simvastatin plus IR consistently retained the $\gamma \mathrm{H} 2 \mathrm{AX}$ foci for $\leq 24 \mathrm{~h}$ after IR in both cell lines, especially in the HCT116 $\mathrm{p}^{-1-}$ cells $(\mathrm{P}<0.001)$. To investigate whether simvastatin affected the DNA repair pathways, we examined the expression levels of homologous recombination $(\mathrm{HR})$ repair proteins $(\operatorname{Rad} 50$, Rad51 and ERCC1) and non-homologous end joining (NHEJ) repair proteins (ku70, ku80 and DNA-PKcs) in HCT116 p53 ${ }^{+/+}$

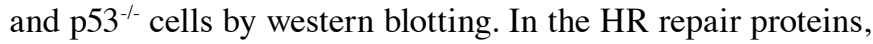
simvastatin decreased the $\operatorname{Rad} 50, \operatorname{Rad} 51$ and ERCC1 protein levels in HCT116 p53 ${ }^{+/+}$and p53 $3^{-/}$cells (Fig. 4B). Furthermore, IR alone significantly increased the expression of the Rad51 protein compared with the control, whereas simvastatin plus
IR reduced the expression of the Rad51 protein compared with IR alone in both cell lines. However, expression of NHEJ repair proteins was not altered by simvastatin in either cell line. These results indicated that simvastatin plus IR prolonged DNA damage and reduced the HR repair protein levels relative to IR alone in HCT116 $\mathrm{p}^{+/+}$and $\mathrm{p} 53^{-/-}$cells; these effects were especially noticeable in HCT116 $\mathrm{p}^{-3^{-/}}$cells.

Simvastatin enhances the radiosensitizing effect by reducing MDM2 expression in p53-deficient cells and xenograft tumors. To investigate the role of simvastatin in the response to radiation according to p53 status, we examined the expression of MDM2-related proteins in HCT116 $\mathrm{p}^{+/+}$and $\mathrm{p} 53^{-/-}$cells and xenograft tumors.

First, we confirmed the p-ATM foci formation, which is an upstream factor of MDM2 in HCT116 $\mathrm{p}^{\mathrm{H}} 3^{+/+}$and $\mathrm{p} 53^{-/-}$cells. Simvastatin plus IR markedly elevated IR-induced p-ATM foci formation compared with IR alone in HCT116 $\mathrm{p} 53^{-/-}$cells $(\mathrm{P}<0.001)$ but not in $\mathrm{p} 53^{+/+}$cells (Fig. $\left.5 \mathrm{~A}\right)$.

We also evaluated the expression of MDM2 and downstream factors of MDM2, including FOXO3a, E-cadherin, and p21, which are tumor suppressor proteins. Regardless of p53 status, simvastatin decreased the MDM2 protein level in both cell lines $(\mathrm{P}<0.001)$. Interestingly, simvastatin strongly increased the expression levels of FOXO3a, E-cadherin, and p21 in HCT116 p53 $3^{-/}$cells but not in p53 ${ }^{+/+}$cells (Fig. 5B). Additionally, simvastatin reduced the MDM2 protein level 
A

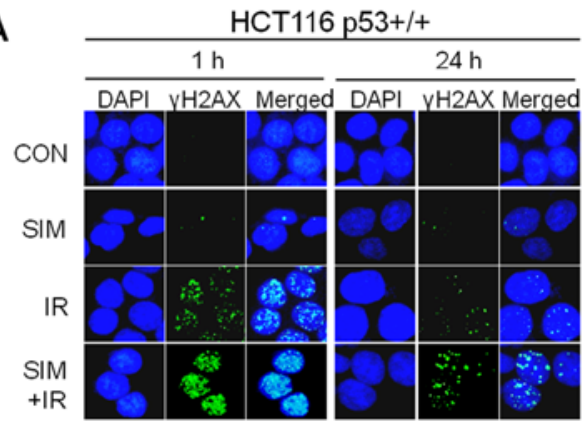

HCT116 p53-/-

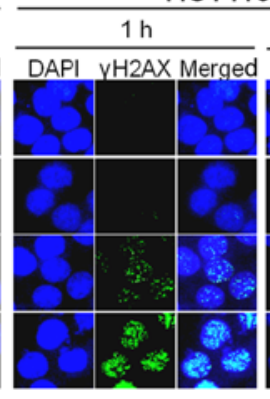

$24 \mathrm{~h}$

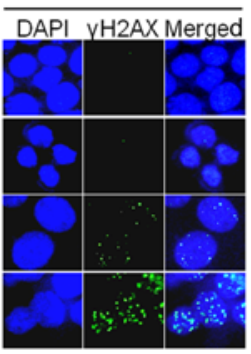

$\stackrel{* * *}{\stackrel{*}{*}}$

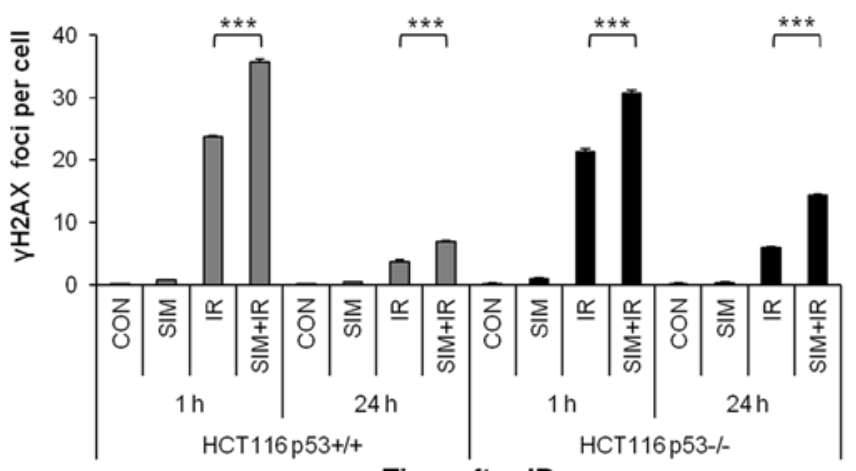

B

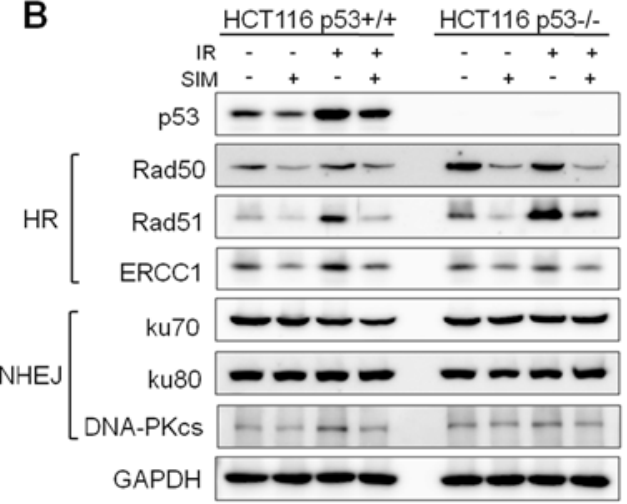

Time after IR

Figure 4. Simvastatin delayed the repair of IR-induced DNA damage in HCT116 p53 $3^{-/}$cells compared with p53 $3^{+/+}$cells. HCT116 p53 ${ }^{+/+}$and p53 pretreated with $5 \mu \mathrm{M}$ simvastatin (SIM) for $24 \mathrm{~h}, 6 \mathrm{~Gy}$ radiation or simvastatin plus IR. (A) To examine the resulting DNA damage, $\gamma \mathrm{H} 2 \mathrm{AX}$ foci formation assays were conducted on HCT116 p53 $3^{+/+}$cells and $\mathrm{p} 53^{-/}$cells, where cells were stained for $\gamma \mathrm{H} 2 \mathrm{AX}$ at 1 and $24 \mathrm{~h}$ after irradiation. Graphs represent the quantification of cells with positive $\gamma \mathrm{H} 2 \mathrm{AX}$ foci, and the bars represent \pm SEM. Statistically significant differences are shown; ${ }^{* * *} \mathrm{P}<0.001$. To investigate the resulting DNA repair, (B) we assessed the expression levels of HR repair proteins (Rad50, Rad51 and ERCC1) and NHEJ repair proteins (ku70, ku80 and DNA-PKcs) in both cell lines by western blotting.

in HCT116 $\mathrm{p}^{-/-}$xenograft tumors $(\mathrm{P}<0.05)$ but not in $\mathrm{p} 53^{+/+}$ xenograft tumors (Fig. 5C). In summary, we demonstrated that simvastatin acted as an MDM2 inhibitor and enhanced the radiosensitivity of $\mathrm{HCT} 116 \mathrm{p} 53^{-/}$cells by increasing p-ATM foci formation and the protein levels of FOXO3a, E-cadherin, and $\mathrm{p} 21$.

\section{Discussion}

Simvastatin, an HMG-CoA reductase inhibitor, exhibits antiproliferative, pro-apoptotic, anti-invasive, and anti-metastatic properties in various cancers. Cancer cells are more sensitive to the statins than normal cells because the levels of HMG-CoA and low-density lipid receptors are elevated in cancer cells (29). Furthermore, simvastatin enhances the radiation responses of various cancer cell types (30) and induces apoptosis through p53-dependent (12) or p53-independent pathways (13). Although numerous studies have suggested that simvastatin may be useful in cancer therapy, the radiosensitizing effect of simvastatin in radioresistant cancer cells remains unelucidated. In this study, we examined the anticancer and radiosensitizing effects of simvastatin in p53-deficient cancer cells, focusing on the underlying mechanisms of the p53-MDM2 interaction.

The anticancer effect of simvastatin on cell growth and cell cycle progression has been studied extensively. Simvastatin reduces cancer cell growth by inducing apoptosis through upregulation of pro-apoptotic proteins (BAX, caspase-3, caspase-8 and caspase-9) and downregulation of anti-apoptotic protein (Bcl-2, Bcl-xl and XIAP) $(3,4,6,8,12,30)$ in the presence or absence of p53. Consistent with previous studies, we found that simvastatin significantly inhibited the proliferation and clonogenic survival of HCT116 p53 wild-type and p53-deficient cells (Fig. 1). This study specifically showed that $\mathrm{p} 53$-deficient cells were more sensitive to simvastatin than HCT116 p53 wild-type cells. Moreover, simvastatin stimulated cell cycle arrest through the reduction of cyclin D1 and survivin in both cell lines, which is consistent with previous reports $(3,12)$. Furthermore, we demonstrated that simvastatin also reduced the expression levels of CDKs (CDK4 and CDK6), cyclin B1, and $\mathrm{pRb}$ in both cell lines (Fig. 3B). One interesting observation in our study was that simvastatin had a new function of reducing HR repair protein levels (Rad50, Rad51 and ERCC1) (Fig. 4B), and simvastatin consequently promoted cancer cell death. These results highlighted the anticancer effects of simvastatin in cancer cells, but the effects did not differ between the HCT116 p53 wild-type and p53-deficient cells.

Previous studies have shown that simvastatin induces G1 arrest (7) and increases IR-induced clonogenic cell death in gastric and colorectal cancer cells, but these studies did not consider p53 status (11). In contrast to these previous studies, we observed that simvastatin did not induce G1 arrest and that simvastatin exhibited radiosensitizing effects only in HCT116 p53-deficient cells. Although simvastatin did not affect the proportion of G1 phase in HCT116 p53 wild-type and p53-deficient cells, simvastatin plus IR induced accumulation of G1 phase by preventing IR-induced G2/M arrest, compared with IR alone; this was especially apparent for HCT116 p53-deficient cells (Fig. 3A). Additionally, simvastatin decreased IR-induced Rad51 and cell cycle regulatory proteins including CDK1, CDK2, cyclin A2, cyclin B1, and 
A

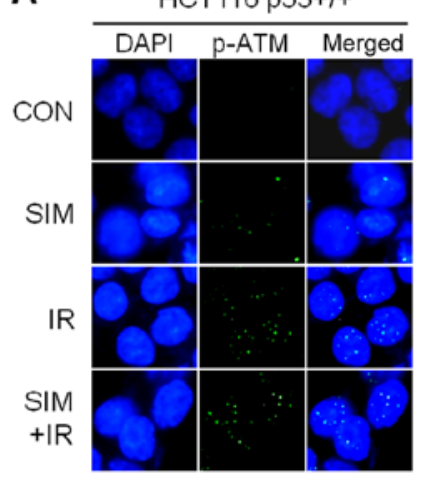

HCT116 p53-/-

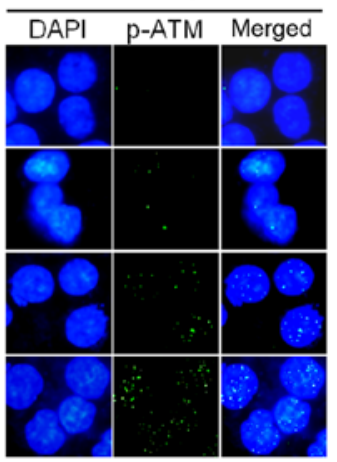

B

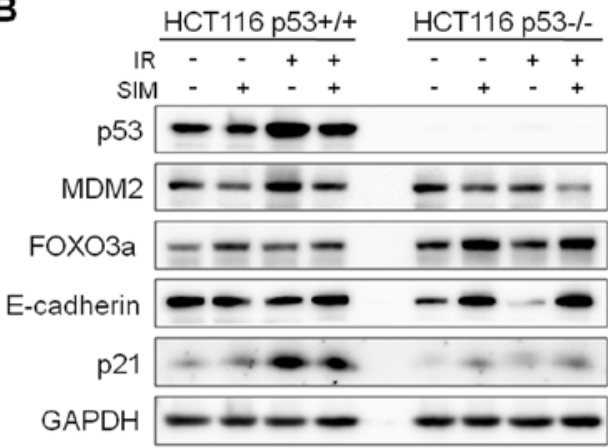

C

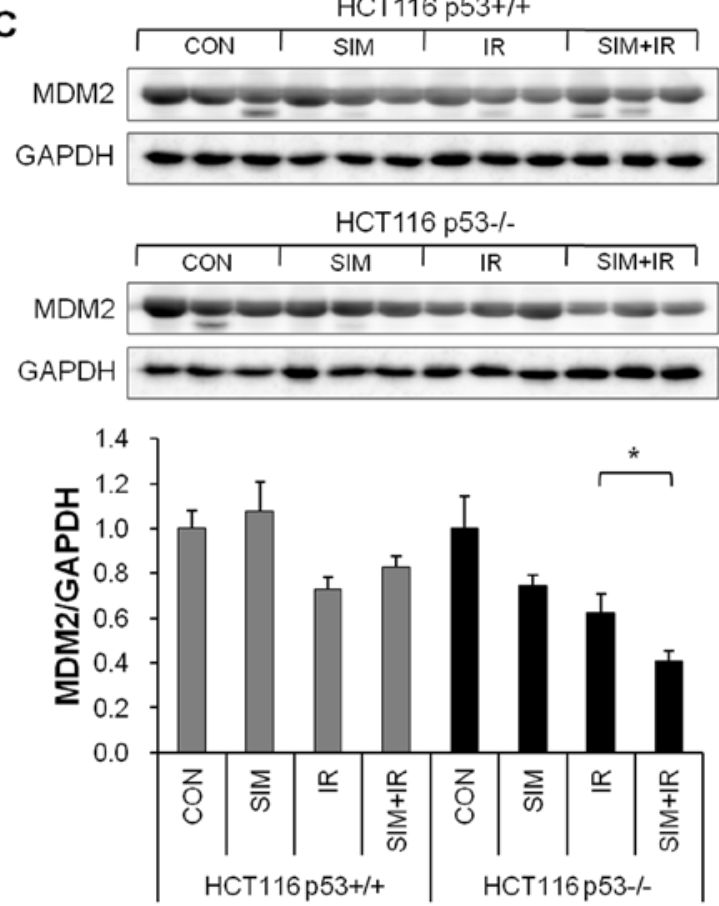

Figure 5. Simvastatin combined with IR attenuates MDM2 protein expression in p53-deficient cells and xenograft tumors. To demonstrate the difference in the simvastatin-mediated radiosensitizing effect between the HCT116 p53 $3^{+/+}$and $\mathrm{p} 53^{-/}$cell lines and xenograft tumors, we focused on the p53-MDM2-related pathway. (A) Both cell lines were treated with $5 \mu \mathrm{M}$ simvastatin (SIM), 6 Gy radiation or both, and $24 \mathrm{~h}$ after IR, immunofluorescence staining was performed for p-ATM. Graphs represent the quantification of cells with positive p-ATM foci. (B) The expression levels of MDM2 and related proteins (FOXO3a, E-cadherin and p21) after treatment of cells with IR for $24 \mathrm{~h}$ were examined by western blotting. (C) After daily treatments with vehicle or simvastatin $\left(20 \mathrm{mg} / \mathrm{kg} /\right.$ day) by intraperitoneal injection for 16 days, HCT116 $553^{+/+}$and $\mathrm{p} 53^{-/-}$tumor tissues were lysed, and the MDM2 protein level was determined by western blotting. Values represent the mean \pm SEM. Statistically significant differences are shown; ${ }^{*} \mathrm{P}<0.05,{ }^{* * * *} \mathrm{P}<0.001$.

survivin in both cell lines (Fig. 3B). Interestingly, although simvastatin did not solely induce DNA damage, simvastatin combined with IR increased IR-induced DNA damage and the duration of $\gamma \mathrm{H} 2 \mathrm{AX}$ foci expression in both cell lines, especially the p53-deficient cell line (Fig. 4A). Collectively, these data suggest that simvastatin-induced radiosensitization in p53-deficient cells may be associated with cell cycle regulation and DNA damage repair pathways.

Based on our data, we examined the mechanism by which simvastatin enhances the anticancer effect and radiation response more strongly in p53-deficient cancer cells than in wild-type cancer cells. MDM2, which is negative regulator of p53, has p53-dependent and p53-independent roles in the regulation of cancer cells $(31,32)$. MDM2 suppresses p53 activities in p53 wild-type cancer cells while it promotes tumor formation and progression in p53-deficient cancer cells (24). When cells are irradiated, IR phosphorylates ATM, sequentially activates p53, and degrades the MDM2 in p53 wild-type cancer cells (33-35), whereas phosphorylated ATM directly reduced MDM2 proteins in p53-deficient cancer cells; but the MDM proteins are consistently present in p53-deficient cancer cells (36). By focusing on the dual actions of the MDM2, we examined the differences of anticancer and radiosensitivity between the p53 wild-type and p53-deficient cells. In this study, we found that simvastatin exhibited a novel function, namely, MDM2 inhibition, regardless of p53 status. After the MDM2 protein reduction by simvastatin, the degraded factors by MDM2 including tumor suppressor proteins FOXO3a, E-cadherin, and p21 was increased, and simvastatin combined with IR increased the formation of p-ATM foci compared with IR alone in p53-deficient but not p53 wild-type cancer cells (Fig. 5). In p53 wild-type cancer, MDM2 inhibition by simvastatin may be specific to activation of $\mathrm{p} 53$, which is a major function of MDM2, and not specific to increasing downstream factors of MDM2. Accordingly, simvastatin inhibited p53-independent functions of MDM2 and thereby promoted 
anticancer effects and radiosensitization of p53-deficient cancer cells.

In conclusion, simvastatin stimulated clonogenic cell death by reducing cell cycle-related proteins and HR DNA repair proteins in p53 wild-type cells and especially in p53-deficient cancer cells. Additionally, simvastatin enhanced the radiation response by reducing the MDM2 proteins levels and inducing the FOXO3a, E-cadherin, and p21 protein levels in p53-deficient cancer cells and xenografts. The exact mechanism of MDM2 inhibition-induced apoptosis and metastasis by simvastatin remains to be clarified. In the present study, we presented the basis for broad therapeutic applicability of simvastatin as an MDM2-based radiosensitizer for the treatment of p53-deficient cancers.

\section{Acknowledgements}

This study was supported by a grant of the Korea Institute of Radiological and Medical Sciences (KIRAMS), funded by Ministry of Science, ICT and Future Planning, Republic of Korea (no. 1711031807/50541-2016).

\section{References}

1. Novac N: Challenges and opportunities of drug repositioning. Trends Pharmacol Sci 34: 267-272, 2013.

2. Ashburn TT and Thor KB: Drug repositioning: Identifying and developing new uses for existing drugs. Nat Rev Drug Discov 3: 673-683, 2004.

3. Shen YY, Yuan Y, Du YY and Pan YY: Molecular mechanism underlying the anticancer effect of simvastatin on MDA-MB-231 human breast cancer cells. Mol Med Rep 12: 623-630, 2015.

4. Hoque A, Chen $\mathrm{H}$ and Xu XC: Statin induces apoptosis and cell growth arrest in prostate cancer cells. Cancer Epidemiol Biomarkers Prev 17: 88-94, 2008.

5. Hindler K, Cleeland CS, Rivera E and Collard CD: The role of statins in cancer therapy. Oncologist 11: 306-315, 2006.

6. Spampanato C, De Maria S, Sarnataro M, Giordano E, Zanfardino M, Baiano S, Cartenì M and Morelli F: Simvastatin inhibits cancer cell growth by inducing apoptosis correlated to activation of Bax and down-regulation of BCL-2 gene expression. Int J Oncol 40: 935-941, 2012.

7. Relja B, Meder F, Wilhelm K, Henrich D, Marzi I and Lehnert M: Simvastatin inhibits cell growth and induces apoptosis and G0/ G1 cell cycle arrest in hepatic cancer cells. Int J Mol Med 26: 735-741, 2010.

8. Huang X, Ma J, Xu J, Su Q and Zhao J: Simvastatin induces growth inhibition and apoptosis in HepG2 and Huh7 hepatocellular carcinoma cells via upregulation of Notch1 expression. Mol Med Rep 11: 2334-2340, 2015.

9. Fang Z, Tang Y, Fang J, Zhou Z, Xing Z, Guo Z, Guo X, Wang W, Jiao W, Xu Z, et al: Simvastatin inhibits renal cancer cell growth and metastasis via AKT/mTOR, ERK and JAK2/STAT3 pathway. PLoS One 8: e62823, 2013.

10. Wang T, Seah S, Loh X, Chan CW, Hartman M, Goh BC and Lee SC: Simvastatin-induced breast cancer cell death and deactivation of PI3K/Akt and MAPK/ERK signalling are reversed by metabolic products of the mevalonate pathway. Oncotarget 7: 2532-2544, 2016.

11. Lim T, Lee I, Kim J and Kang WK: Synergistic effect of simvastatin plus radiation in gastric cancer and colorectal cancer: Implications of BIRC5 and connective tissue growth factor. Int J Radiat Oncol Biol Phys 93: 316-325, 2015.

12. Wang Y, Xu SL, Wu YZ, Zhao MS, Xu WJ, Yang HY and Li YX: Simvastatin induces caspase-dependent apoptosis and activates P53 in OCM-1 cells. Exp Eye Res 113: 128-134, 2013.
13. Koyuturk M, Ersoz M and Altiok N: Simvastatin induces apoptosis in human breast cancer cells: p53 and estrogen receptor independent pathway requiring signalling through JNK. Cancer Lett 250: 220-228, 2007.

14. Kumar S: Second malignant neoplasms following radiotherapy. Int J Environ Res Public Health 9: 4744-4759, 2012.

15. Klein HL: The consequences of Rad51 overexpression for normal and tumor cells. DNA Repair (Amst) 7: 686-693, 2008.

16. Rohwer $\mathrm{N}$ and Cramer T: Hypoxia-mediated drug resistance: Novel insights on the functional interaction of HIFs and cell death pathways. Drug Resist Updat 14: 191-201, 2011.

17. El-Deiry WS: The role of p53 in chemosensitivity and radiosensitivity. Oncogene 22: 7486-7495, 2003.

18. Böhnke A, Westphal F, Schmidt A, El-Awady RA and Dahm-Daphi J: Role of p53 mutations, protein function and DNA damage for the radiosensitivity of human tumour cells. Int J Radiat Biol 80: 53-63, 2004.

19. Gudkov AV and Komarova EA: The role of p53 in determining sensitivity to radiotherapy. Nat Rev Cancer 3: 117-129, 2003.

20. Alarcon-Vargas D and Ronai Z: p53-Mdm2 - the affair that never ends. Carcinogenesis 23: 541-547, 2002.

21. Meek DW: Tumour suppression by p53: A role for the DNA damage response? Nat Rev Cancer 9: 714-723, 2009.

22. Ganguli $\mathrm{G}$ and Wasylyk B: p53-independent functions of MDM2. Mol Cancer Res 1: 1027-1035, 2003.

23. Zhang Z and Zhang R: p53-independent activities of MDM2 and their relevance to cancer therapy. Curr Cancer Drug Targets 5: 9-20, 2005.

24. Rayburn E, Zhang R, He J and Wang H: MDM2 and human malignancies: Expression, clinical pathology, prognostic markers, and implications for chemotherapy. Curr Cancer Drug Targets 5: 27-41, 2005.

25. Feng FY, Zhang Y, Kothari V, Evans JR, Jackson WC, Chen W, Johnson SB, Luczak C, Wang S and Hamstra DA: MDM2 inhibition sensitizes prostate cancer cells to androgen ablation and radiotherapy in a p53-dependent manner. Neoplasia 18: 213-222, 2016.

26. Zhang Z, Wang H, Li M, Agrawal S, Chen X and Zhang R: MDM2 is a negative regulator of p21WAF1/CIP1, independent of p53. J Biol Chem 279: 16000-16006, 2004.

27. Zhang Z, Wang H, Prasad G, Li M, Yu D, Bonner JA, Agrawal S and Zhang R: Radiosensitization by antisense anti-MDM2 mixed-backbone oligonucleotide in in vitro and in vivo human cancer models. Clin Cancer Res 10: 1263-1273, 2004.

28. Duong HQ, You KS, Oh S, Kwak SJ and Seong YS: Silencing of NRF2 reduces the expression of ALDH1A1 and ALDH3A1 and sensitizes to 5-FU in pancreatic cancer cells. Antioxidants 6: 6, 2017.

29. Liao JK: Isoprenoids as mediators of the biological effects of statins. J Clin Invest 110: 285-288, 2002.

30. Hwang KE, Na KS, Park DS, Choi KH, Kim BR, Shim H, Jeong ET and Kim HR: Apoptotic induction by simvastatin in human lung cancer A549 cells via Akt signaling dependent down-regulation of survivin. Invest New Drugs 29: 945-952, 2011.

31. Shi D and Gu W: Dual roles of MDM2 in the regulation of p53: Ubiquitination dependent and ubiquitination independent mechanisms of MDM2 repression of p53 activity. Genes Cancer 3: 240-248, 2012.

32. Li Q and Lozano G: Molecular pathways: Targeting Mdm2 and Mdm4 in cancer therapy. Clin Cancer Res 19: 34-41, 2013.

33. Chen L, Gilkes DM, Pan Y, Lane WS and Chen J: ATM and Chk2-dependent phosphorylation of MDMX contribute to p53 activation after DNA damage. EMBO J 24: 3411-3422, 2005.

34. Perry ME: Mdm2 in the response to radiation. Mol Cancer Res 2: 9-19, 2004

35. de Toledo SM, Azzam EI, Dahlberg WK, Gooding TB and Little JB: ATM complexes with HDM2 and promotes its rapid phosphorylation in a p53-independent manner in normal and tumor human cells exposed to ionizing radiation. Oncogene 19: 6185-6193, 2000.

36. Perry ME: The regulation of the p53-mediated stress response by MDM2 and MDM4. Cold Spring Harb Perspect Biol 2: a000968, 2010. 